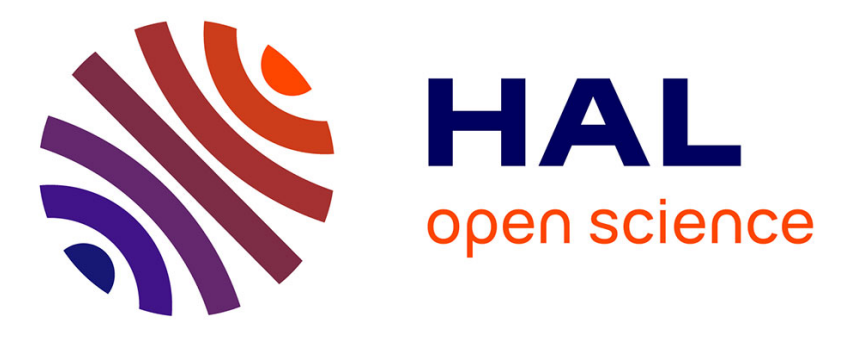

\title{
Microstructure and heat treatment effect on transformation strain in steels: part 2 modelling
} Takayuki Otsuka, Daisuke Satani, Kenji Yamamoto, Kazuo Okamura, Renald Brenner, Brigitte Bacroix

\section{- To cite this version:}

Takayuki Otsuka, Daisuke Satani, Kenji Yamamoto, Kazuo Okamura, Renald Brenner, et al.. Microstructure and heat treatment effect on transformation strain in steels: part 2 modelling. Materials Science and Technology, 2018, 35 (2), pp.187-194. 10.1080/02670836.2018.1548111 . hal-02188682

\section{HAL Id: hal-02188682 \\ https://hal.sorbonne-universite.fr/hal-02188682}

Submitted on 18 Jul 2019

HAL is a multi-disciplinary open access archive for the deposit and dissemination of scientific research documents, whether they are published or not. The documents may come from teaching and research institutions in France or abroad, or from public or private research centers.
L'archive ouverte pluridisciplinaire HAL, est destinée au dépôt et à la diffusion de documents scientifiques de niveau recherche, publiés ou non, émanant des établissements d'enseignement et de recherche français ou étrangers, des laboratoires publics ou privés. 


\title{
Microstructure and heat treatment effect on transformation strain in steels: part 2 modelling
}

\author{
Takayuki Otsuka (1) ${ }^{a}$, Daisuke Satani ${ }^{a}$, Kenji Yamamoto ${ }^{b}$, Kazuo Okamura (1) ${ }^{a}$, Renald Brenner ${ }^{c}$ and \\ Brigitte Bacroix (1) d

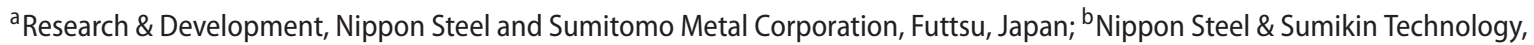

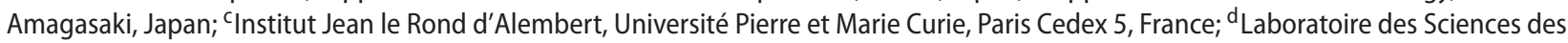 \\ Procédés et des Matériaux, Villetaneuse, France
}

\begin{abstract}
Anisotropic dilatation during phase transformation in steels can be caused by banded microstructure. As well as the directionality in mechanical properties, the anisotropic transformation strain can result in distortion and dimension change of the products. Hence, mechanical assessment of this phenomenon is of great importance. In this paper, a numerical approach using Crystal Plasticity Fast Fourier Transform (CPFFT) has been developed and used, reproducing the anisotropic transformation stain during heating (experimental results are reported in Part 1). It was confirmed by the numerical simulation that the banded microstructure in microstructure can be one of the most important reasons for the anisotropy of transformation strain.
\end{abstract}

\section{KEYWORDS}

Transformation strain; anisotropic dilatation; directional strain; band structure; pearlite band; crystal plasticity; FFT; CPFFT

\section{Introduction}

Recent developments in numerical simulations of heat treatment process of steels achieved precise prediction of distortion and residual stress [1-4]. This can be realised by considering phase transformation effect transformation strain and transformation plastic strain - as well as the classical thermo-mechanical coupling. In this case, transformation strain is usually supposed as isotropic. However, materials having banded structure (e.g. ferrite-pearlite band structure) sometimes exhibit anisotropic strain during phase transformation [5-7]. According to the results by Kop et al. [5], the total transformation strain in normal direction is 0.0050 whereas 0.0029 in rolling direction. Together with the transformation plasticity, which is same order as the transformation strain [8], the anisotropy in transformation strain during cooling is considered to be one of the important factors affecting the distortion and residual stress in the work after heat treatment. Therefore, predicting the anisotropy caused by the heterogeneous microstructure is of great importance in order to reveal the mechanism of anisotropic transformation strain observed with banded structure. In this respect, the aim of this study is defined to develop a full-field numerical model with the effect of banded microstructure. In this numerical model, a classical crystal plasticity constitutive model is employed, which is extended to comprise the transformation strain, to calculate the macroscopic (overall) transformation strain behaviour and reveal the microstructure effect.
Mechanical behaviour of steels during phase transformation has received much attention and many simulation works have been dedicated to the complicated problem [8-12] and a more macroscopic application was made by Mackerle [13]. Most of the previous numerical works have used the finite element method. In contrast to the finite element method, Fast Fourier Transform (FFT) numerical scheme has recently proved its efficiency to solve periodic boundary elastoplastic problems, which is firstly introduced by Moulinec and Suquet [14]. This method was applied to the crystal plasticity problems (CPFFT) $[15,16]$ and the mechanical behaviour associated with phase transformation called transformation plasticity by one of the authors [17]. Thus in this paper, CPFFT is introduced and used to a qualitative investigation of the band structure effect on the anisotropic dilatation during phase transformation, which is to be contrasted to the case with homogeneous microstructure.

\section{Numerical approach}

A CPFFT numerical scheme is used in this study to reveal mechanical behaviour during phase transformation. The FFT application for stress-strain calculation uses Green's functions method to solve a periodic boundary value problem for heterogeneous media. This method was extended by Lee et al. [18] and Lebensohn et al. [19] to application of crystal plasticity analyses. Stress distribution caused by heterogeneous 
transformation strain is considered in the previous work [15] and this model is to be a further application of the anisotropic $\alpha \rightarrow \gamma$ transformation strain behaviour in ferrite-pearlite band structured media. In the following sections, formulae in the framework of CPFFT are briefly introduced and calculation condition together with results and discussions are demonstrated.

\section{Model description}

\section{FFT formalism for an elastoplastic problem}

Let the displacement vector be divided into uniform value and fluctuation term as:

$$
u(x)=u^{\prime}(x)+\bar{\varepsilon} x,
$$

where $\boldsymbol{u}^{\prime}(\boldsymbol{x})$ is the fluctuation term of periodic displacement. Then, the strain field reads:

$$
\boldsymbol{\varepsilon}(\boldsymbol{u}(\boldsymbol{x}))=\boldsymbol{\varepsilon}\left(\boldsymbol{u}^{\prime}(\boldsymbol{x})\right)+\bar{\varepsilon},
$$

with $\boldsymbol{\varepsilon}\left(\boldsymbol{u}^{\prime}(\boldsymbol{x})\right)=0$.

A rate form of elastoplastic constitutive equation considering transformation strain can be written as:

$\dot{\boldsymbol{\sigma}}(\boldsymbol{x})=\boldsymbol{C}(\boldsymbol{x}): \dot{\boldsymbol{\varepsilon}}^{e}(\boldsymbol{x})=\boldsymbol{C}(\boldsymbol{x}):\left(\dot{\boldsymbol{\varepsilon}}(\boldsymbol{x})-\dot{\boldsymbol{\varepsilon}}^{p}(\boldsymbol{x})-\dot{\boldsymbol{\varepsilon}}^{m}(\boldsymbol{x})\right)$,

where $\boldsymbol{C}(x)$ is the elastic tensor, $\dot{\boldsymbol{\varepsilon}}^{e}, \dot{\boldsymbol{\varepsilon}}, \dot{\boldsymbol{\varepsilon}}^{p}$ and $\dot{\boldsymbol{\varepsilon}}^{m}$ are elastic, total, plastic and transformation strain rate tensors respectively. Here, the polarisation tensor $\dot{\boldsymbol{\tau}}(\boldsymbol{x})$ and the elastic tensor $C^{0}$ of the reference media are introduced to consider the heterogeneity. We obtain (\# represents periodic boundary condition):

$$
\begin{aligned}
& \dot{\boldsymbol{\sigma}}(\boldsymbol{x})=C^{0}: \dot{\boldsymbol{\varepsilon}}(\boldsymbol{x})+\left(\boldsymbol{C}(\boldsymbol{x})-C^{0}\right): \dot{\boldsymbol{\varepsilon}}(\boldsymbol{x}) \\
& \quad+\boldsymbol{C}(\boldsymbol{x}):\left(\dot{\boldsymbol{\varepsilon}}^{p}(\boldsymbol{x})+\dot{\boldsymbol{\varepsilon}}^{m}(\boldsymbol{x})\right)=C^{0}: \dot{\boldsymbol{\varepsilon}}(\boldsymbol{x})+\dot{\boldsymbol{\tau}}(\boldsymbol{x}), \\
& \forall x \in V, \operatorname{div} \dot{\boldsymbol{\sigma}}=0 \forall x \in V, \dot{\boldsymbol{u}} \#, \dot{\boldsymbol{\sigma}} \cdot \boldsymbol{n}-\# .
\end{aligned}
$$

Solution of the problem reads:

$$
\dot{\boldsymbol{\varepsilon}}(\boldsymbol{x})=\dot{\bar{\varepsilon}}-\Gamma^{0} * \dot{\boldsymbol{\tau}} .
$$

Equations (4) in Fourier space is:

$$
\widehat{\dot{\sigma}}(\xi)=\mathrm{i} C^{0}:\left(\widehat{\boldsymbol{u}}^{\prime}(\xi) \otimes \xi\right)+\widehat{\dot{\boldsymbol{\tau}}}(\xi), \hat{\mathrm{i}} \widehat{\dot{\sigma}}(\xi) \cdot \xi=0,
$$

where, $\xi$ is frequency, and the non-italic character i represents imaginary number. Eliminating $\widehat{\hat{\sigma}}$ from Equations (6) gives

$$
\widehat{\ddot{u}}^{\prime}(\xi)=\frac{\mathrm{i}}{2}\left(\boldsymbol{N}^{0} \otimes \xi+\xi \otimes N^{0}\right) \widehat{\dot{\boldsymbol{\tau}}}(\xi),
$$

where

$$
\begin{gathered}
N^{0}(\xi)=K^{0}(\xi)^{-1}, K^{0}(\xi)=C^{0}:(\xi \otimes \xi), \\
\widehat{\dot{\varepsilon}}(\xi)=\frac{i}{2}\left(\xi \otimes \widehat{\boldsymbol{u}}^{\prime}(\xi)+\widehat{\boldsymbol{u}}^{\prime}(\xi) \otimes \xi\right)=-\hat{\boldsymbol{\Gamma}}^{0}(\xi): \widehat{\dot{\boldsymbol{\tau}}}(\xi),
\end{gathered}
$$

$$
\hat{\boldsymbol{\Gamma}}^{0}(\boldsymbol{\xi})=\frac{1}{4}\left(N_{l i}^{0} \xi_{j} \xi_{k}+N_{k i}^{0} \xi_{j} \xi_{l}+N_{l j}^{0} \xi_{i} \xi_{k}+N_{k j}^{0} \xi_{i} \xi_{l}\right) .
$$

$\hat{\boldsymbol{\Gamma}}^{0}$ is the periodic Green's operator. According to these formulations, strain rate can be calculated by taking inverse Fourier transform of Equation (9) and stress rate is obtained by constitutive equation shown in Equation (3).

\section{Elastoplastic constitutive equations (crystal plasticity)}

Classical crystal plasticity framework for numerical analysis has been introduced and extended by [20-24]. Along with these works, crystal plasticity constitutive equations are demonstrated by follows. Plastic strain rate caused by dislocation glide on slip systems is a summation of slip rate $\dot{\gamma}^{\alpha}$ on each slip system $\alpha$, such that:

$$
\dot{\boldsymbol{\varepsilon}}^{p}=\sum_{\alpha} \boldsymbol{p}^{\alpha} \dot{\gamma}^{\alpha}
$$

where $\boldsymbol{p}^{\alpha}$ is the Schmid tensor of $\alpha$ slip system.

$$
\boldsymbol{p}^{\alpha}=\frac{1}{2}\left(\boldsymbol{s}^{\alpha} \otimes \boldsymbol{m}^{\alpha}+\boldsymbol{m}^{\alpha} \otimes \boldsymbol{s}^{\alpha}\right),
$$

where $\boldsymbol{s}^{\alpha}$ and $\boldsymbol{m}^{\alpha}$ are respectively the slip direction and slip plane normal of the $\alpha$ slip system. Slip system is active, i.e. plastic deformation takes place, when resolved shear stress $\tau^{\alpha}$ on the slip plane equals to the Critical Resolved Shear Stress (CRSS) $g^{\alpha}$, such that:

$$
\left|\tau^{\alpha}\right|=\left|p^{\alpha}: \sigma\right|=g^{\alpha} .
$$

The viscoplastic constitutive model has been developed [22-26] and since then many applications have been made [27-29]. According to this method, all slip systems are active and their deformation magnitude is controlled by the strain rate sensitivity exponent. For relatively low-temperature range (elastoplastic regime), small strain rate sensitivity exponent value should be chosen whereas for relatively hightemperature range (elasto-viscoplastic regime) large value should be chosen. By taking extremely small strain rate sensitivity exponent, one can reproduce the pseudo elasto-plastic constitutive relation. However, the small strain rate sensitivity exponent sometimes makes the calculation unstable even though the stabilisation numerical technique [23] is employed. In this case, enough small increment should be taken resulting in much consumption of computational resources and extremely long calculation time. For this reason, rate-independent model proposed by Hutchinson [30] is more efficient to reproduce pure elasto-plastic regime and conserve more computation time. In this method, the maximum number of active slip systems is limited to 5. However, the model provides accurate solutions for the elasto-plastic problems with phase transformation such as transformation plasticity [17]. Thus, this 
research uses the following rate-independent solution for determining the slip rate of each slip system by using the consistence condition:

$$
\dot{\tau}^{\alpha}=\operatorname{sgn}\left(\tau^{\alpha}\right) \dot{g}^{\alpha} .
$$

The hardening law is simply defined here as:

$$
\dot{g}^{\alpha}=\sum_{\beta} h^{\alpha \beta}\left|\dot{\gamma}^{\beta}\right|
$$

with $h^{\alpha \beta}$ the hardening coefficients. The phenomenological expression of hardening parameter can be written as [22]:

$$
\begin{gathered}
h^{\alpha \alpha}=h=H_{0} \operatorname{sech}^{2}\left[\frac{H_{0} \sum_{\beta} \gamma^{\beta}}{\tau_{s}-\tau_{0}}\right], \\
h^{\alpha \beta}=q h+(1-q) h \delta_{\alpha \beta},
\end{gathered}
$$

where $H_{0}$ is the hardening modulus at initial yield, $\tau_{0}$ is the initial yield stress value and $\tau_{s}$ is the saturated stress value. The parameter $q$ is the ratio of self-hardening and latent-hardening.

By using the constitutive laws, one obtains:

$$
\begin{gathered}
\dot{\boldsymbol{\sigma}}: \boldsymbol{p}^{\alpha}=\boldsymbol{C}:\left(\dot{\boldsymbol{\varepsilon}}-\dot{\boldsymbol{\varepsilon}}^{p}-\dot{\boldsymbol{\varepsilon}}^{m}\right): \boldsymbol{p}^{\alpha}=\boldsymbol{C}:\left(\dot{\boldsymbol{\varepsilon}}-\dot{\boldsymbol{\varepsilon}}^{m}\right): \boldsymbol{p}^{\alpha} \\
\quad-\sum_{\beta} \boldsymbol{p}^{\alpha}: \boldsymbol{C}: \boldsymbol{p}^{\beta} \dot{\gamma}^{\beta}=\sum_{\beta} h^{\alpha \beta} \dot{\gamma}^{\beta} .
\end{gathered}
$$

Solving Equation (18) yields slip rate of each slip system, such that:

$$
\dot{\gamma}^{\alpha}=\boldsymbol{f}^{\alpha}:\left(\dot{\boldsymbol{\varepsilon}}-\dot{\boldsymbol{\varepsilon}}^{m}\right) \text { and } \boldsymbol{f}^{\alpha}=\sum_{\beta} Y^{\alpha \beta} \boldsymbol{C}: \boldsymbol{p}^{\beta},
$$

where

$$
Y^{\alpha \beta}=\left(X^{\alpha \beta}\right)^{-1} \text { and } X^{\alpha \beta}=h^{\alpha \beta}+\boldsymbol{p}^{\alpha}: C: \boldsymbol{p}^{\beta} \text {. }
$$

To make the matrix $X^{\alpha \beta}$ being non-singular, the maximum number of active slip systems, i.e. the rank of the matrix $X^{\alpha \beta}$ is limited to 5 .

\section{Calculation condition}

Initial microstructure comprises 200 grains of ferrite phase in its representative volume element (RVE) which are made by using Voronoi Tessellation technique and each grain is assigned its crystallographic orientation. Figure 1 shows the initial microstructure in which each grain has its unique colour. In the RVE, $128^{3}$ regular material points are fixed and the constitutive relationship is defined according to its Euler angle. For the case with banded microstructure (heterogeneous case), layers of pearlite are initially defined in the RVE as shown in Figure 1, which corresponds to the microstructure of $0.15 \%$ carbon-manganese steel (CMn2 grade of part 1). The nucleation site of austenite grain seeds are randomly spread inside the RVE and

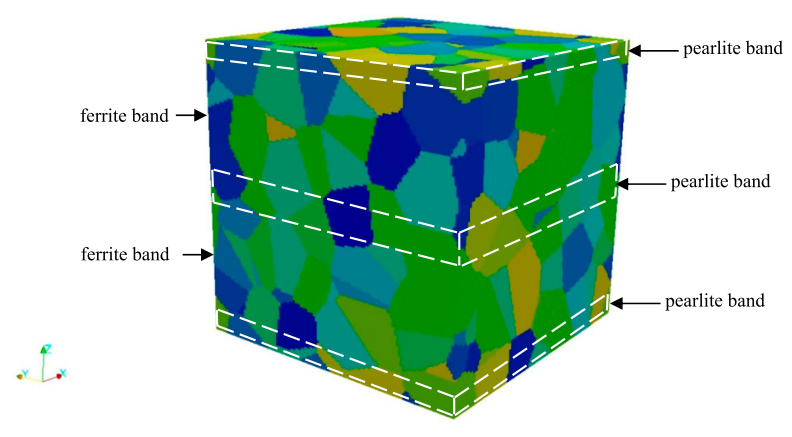

Figure 1. Initial microstructure ma de by Voronoi tessellation method (200 grains) - ferrite and pearlite bands are defined in the case of the heterogeneous microstructure.

the nucleation is defined to take place simultaneously inside the pearlite bands. After the nucleation of the austenite phase, the austenite phase grows isotropically keeping the spherical shape until they encounter the other transformed austenite grains. When the pearlite decomposition is finished, then the ferrite bands initiate austenite phase transformation. In contrast to the banded microstructure case, the more homogeneous transformation is realised by simultaneous nucleation throughout the RVE and uniform growth of the austenite phase. In conjunction with the evolution of phase transformation, a microscopic isotropic transformation strain occurs due to the density difference between the mother and daughter phase. The microscopic transformation strain, defined in the following equation, is induced within one calculation step $(\boldsymbol{t} \rightarrow \boldsymbol{t}+\boldsymbol{\Delta t})$ when phase transformation at each calculation grid takes place:

$$
\int_{t}^{t+\Delta t} \dot{\boldsymbol{\varepsilon}}^{m} \mathrm{~d} t=\beta 1
$$

where $\beta$ is the total transformation strain set by $\beta=$ $-4.55 \times 10^{-3}$ in this study. For the simplicity, the mechanical behaviours for ferrite and pearlite are used, which are slightly softer than that of austenite. The parameters used in this study for crystal plasticity analyses are summarised in Table 1. In addition to the incorporation of the microscopic transformation strain, the set of slip systems of initial phase (bcc -48 slip systems, $\{110\}<111>,\{112\}<111>,\{123\}<111>)$ is replaced by the set of fcc slip systems (fcc - 12 slip systems, $\{100\}<011>$ ) and at the same time, cumulative plastic strain is reset as well as all the other parameters appear in the constitutive equations are set to those of initial daughter phase.

Table 1. Parameters for crystal plasticity calculation.

\begin{tabular}{lcc}
\hline parameters & Ferrite and pearlite $(\alpha)$ & Austenite $(\gamma)$ \\
\hline$\tau_{0}$ & 20.0 & 22.3 \\
$\tau_{s}$ & 38.0 & 40.0 \\
$H_{0}$ & 10.0 & 50.0 \\
$q$ & 1.0 & 1.0 \\
\hline
\end{tabular}




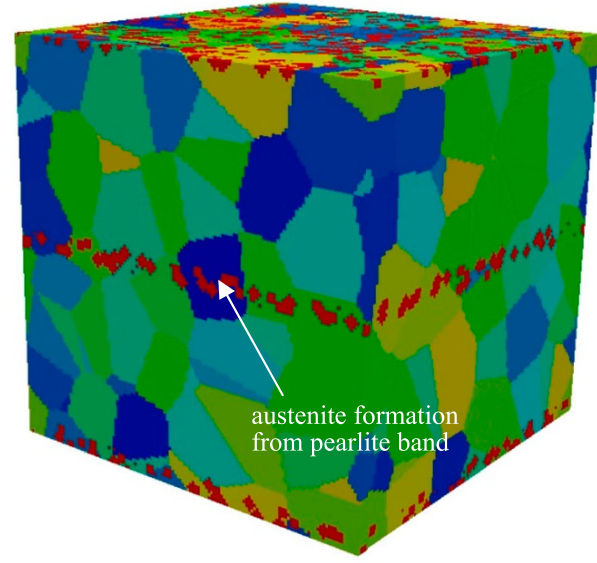

(a) $1 \%$ transformed

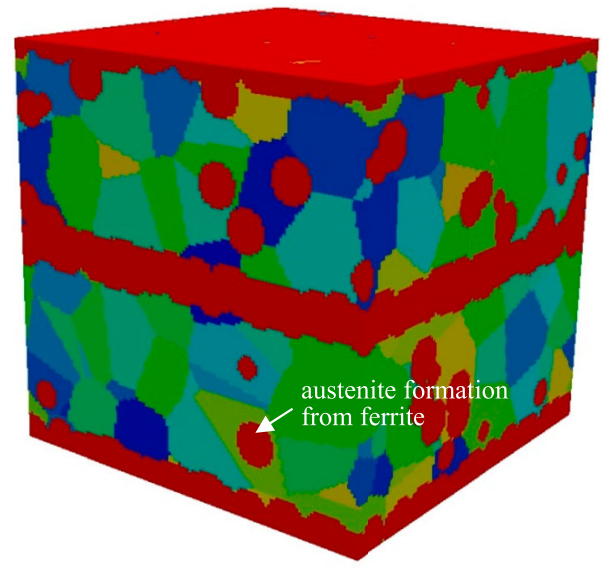

(c) $20 \%$ transformed

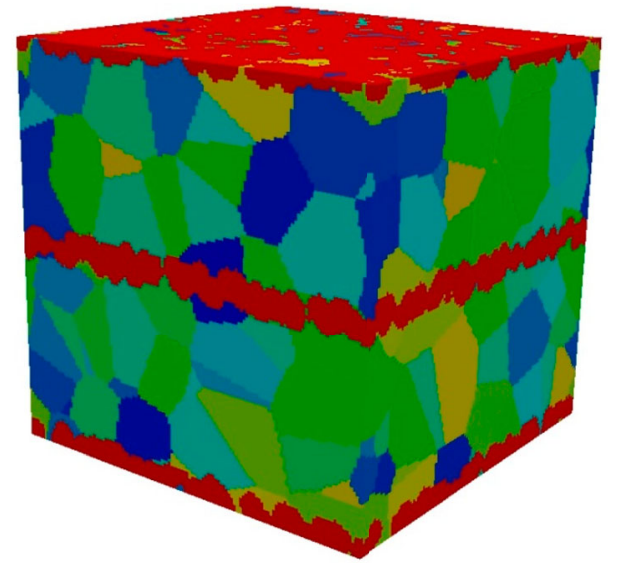

(b) $7 \%$ transformed

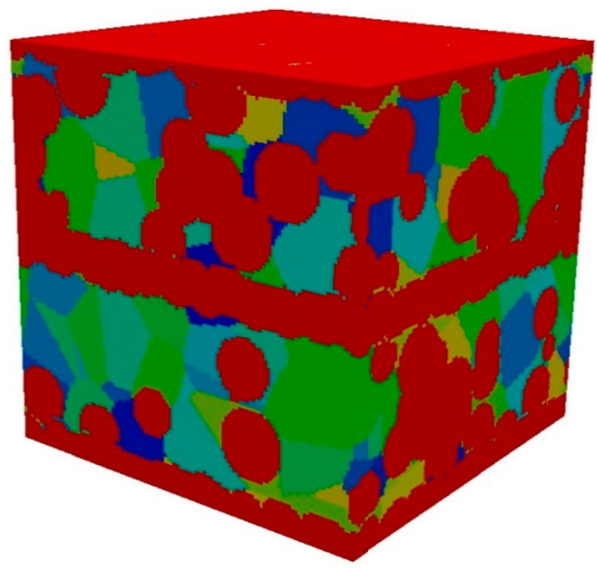

(d) $55 \%$ transformed

Figure 2. Transformed fraction (austenite phase in red colour). (a) $1 \%$ transformed. (b) $7 \%$ transformed. (c) $20 \%$ transformed. (d) $55 \%$ transformed

The transformation strain is the key driving agent for the stress evolution as the elasto-plastic strain occurs accommodating the transformation strain. In each step, macroscopic (average value in the RVE) strain and stress are calculated.

Note that the displacement, strain (and thus stress) and microstructure are periodically reflecting the FFT formulation restriction.

\section{Results and discussions}

\section{Heterogeneous case}

The crystal plasticity calculation is conducted starting with the pearlite band decomposition into austenite phase followed by ferrite phase decomposition as depicted in Figure 2. When volume fraction of austenite phase reaches $7-20 \%$, almost whole region of pearlite band is transformed. At this stage, the ferrite decomposition initiates. The transformed fraction has a smaller volume than the original phase and thus the strain incompatibility occurs which results in the dislocation glide on slip systems. The effect of the volume change can be clearly seen in the equivalent plastic strain distribution shown in Figure 3. More precisely, the plastic strain accumulation is more pronounced at the vicinity of transformation boundaries as is depicted in Figure 3(b,c). Note that the cumulative plastic strain is reset along with the phase transformation reflecting the initialisation of work hardening behaviour; this is the primary reason for the plastic strain decreases inside the crust of the daughter phase. This potentially causes the enhancement of plastic strain called transformation plasticity when external stress is applied $[8,17,31]$.

The macroscopic strain (average strain in the RVE) for each direction is calculated during the transformation process and is shown in Figure 4. At the early stage of phase transformation, i.e. during pearlite band decomposition, strain evolution in z-direction (perpendicular to the pearlite band plane), larger change in strain in z-direction is found more than in other two directions reflecting the geometrical heterogeneity of pearlite band structure. The mechanistic nature of the anisotropic dilatation during pearlite decomposition can be explained as follows. When 


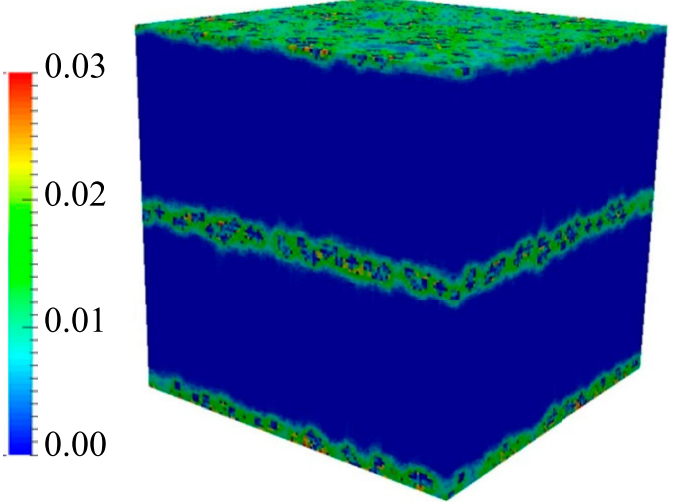

(a) $1 \%$ transformed

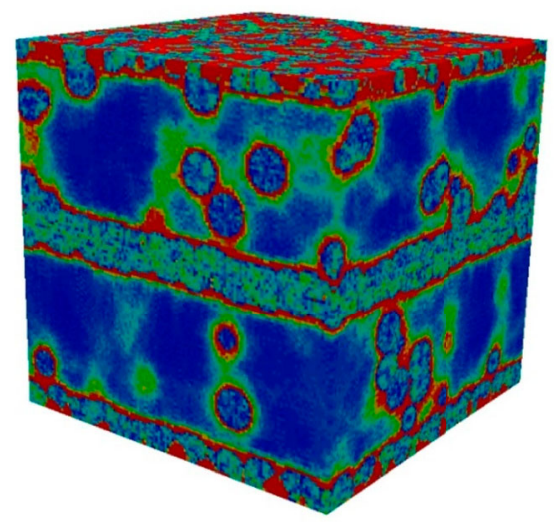

(c) $20 \%$ transformed

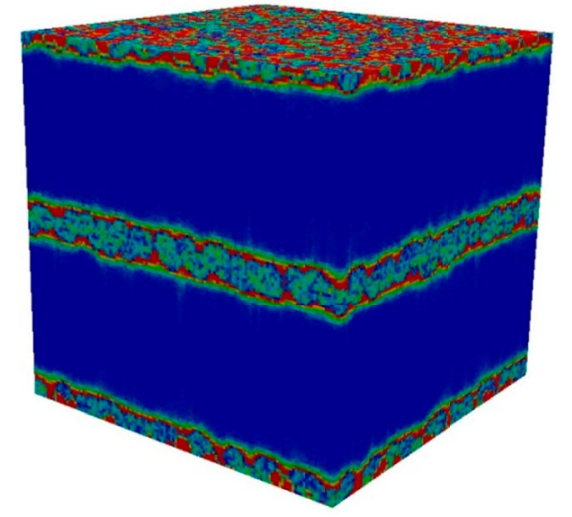

(b) $7 \%$ transformed

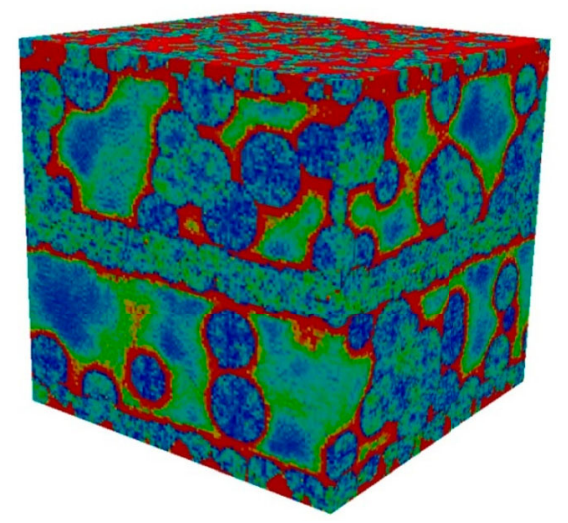

(d) $55 \%$ transformed

Figure 3. Equivalent plastic strain distribution. (a) $1 \%$ transformed. (b) $7 \%$ transformed. (c) $20 \%$ transformed. (d) $55 \%$ transformed

pearlite band is transformed into austenite, microscopic isotropic volume shrinkage occurs. However, the deformation (in $\mathrm{x}-\mathrm{y}$ plane) in transforming pearlite bands is restricted by the ferrite bands and thus the plastic deformation in $\mathrm{z}$ plane is likely to take place rather than in $\mathrm{x}$-y plane because less mechanical restriction exists in $\mathrm{z}$ direction. This results in larger transformation strain in $\mathrm{z}$-direction than in other directions. As austenite phase is harder than the ferrite phase, this effect is still true for the ferrite decomposition stage. The austenite bands, which were initially pearlitic, block the deformation of the ferrite bands in $\mathrm{x}-\mathrm{y}$ plane during ferrite decomposition. Therefore, during ferrite $\rightarrow$ austenite phase transformation, again the strain change in $\mathrm{z}$-direction is more pronounced than in other directions, which can also be interpreted from Figure 4. A qualitative agreement is found here between numerical analyses and what was measured by the experiment (in Part 1). Above mechanisms drawn from the numerical results coincides very well with the postulate put forward by Kop et al. [5].

\section{Homogeneous case}

In this section, the homogeneous phase transformation is simulated. An assumption is made here that the initial ferrite-pearlite phase is homogeneous and no

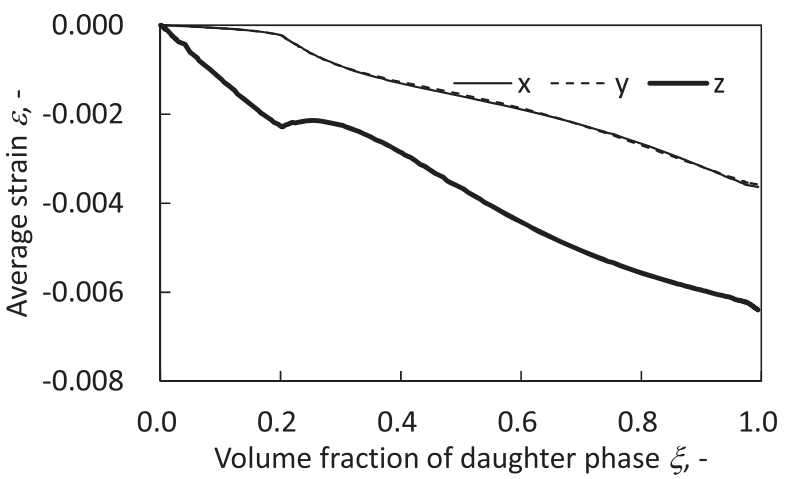

Figure 4. Evolution of equivalent plastic strain during phase transformation (heterogeneous case).

distinction between ferrite and pearlite phase is supposed. In this case, austenite grains in the RVE follow simultaneous nucleation and isotropic grain growth that is demonstrated by the simulation results in the red coloured austenite grains in Figure 5.

Along with the heterogeneous case, distribution of equivalent plastic strain for the homogeneous case is exhibited in Figure 6. Compared with the heterogeneous case, remarkable plastic strain accumulation is observed in daughter phase (Figure 6(d)) due to stronger constraint for the heterogeneous case than for the homogeneous case. This reasoning attributes the 


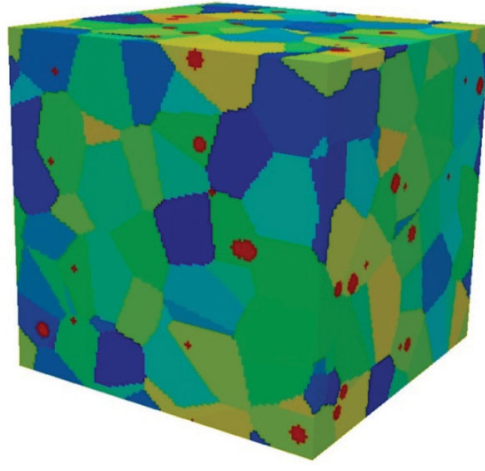

(a) $1 \%$ transformed

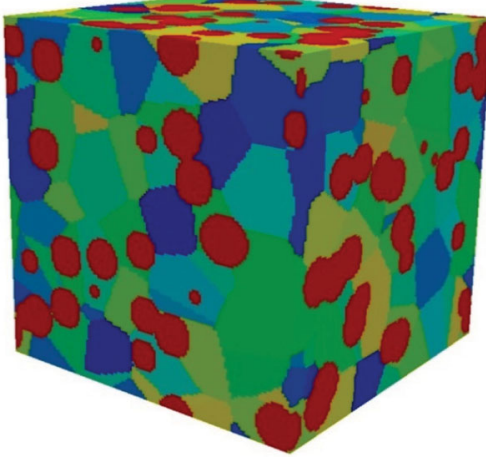

(c) $20 \%$ transformed

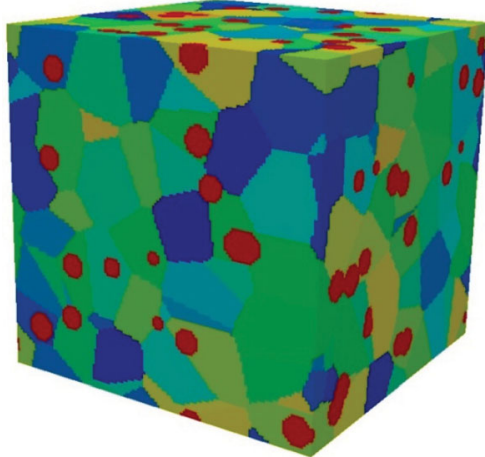

(b) $7 \%$ transformed

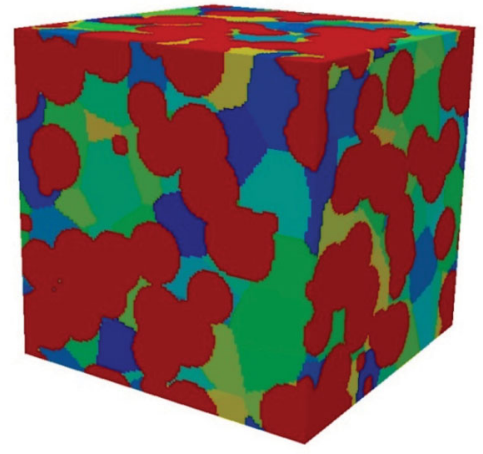

(d) $55 \%$ transformed

Figure 5. Transformed fraction (austenite phase in red colour). (a) $1 \%$ transformed. (b) $7 \%$ transformed. (c) $20 \%$ transformed. (d) $55 \%$ transformed

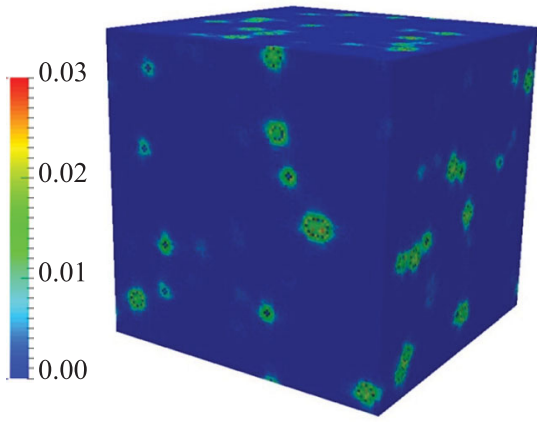

(a) $1 \%$ transformed

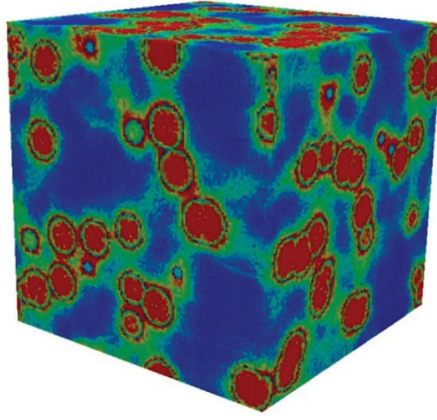

(c) $20 \%$ transformed

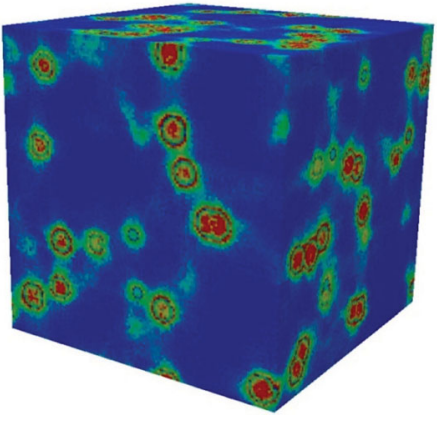

(b) $7 \%$ transformed

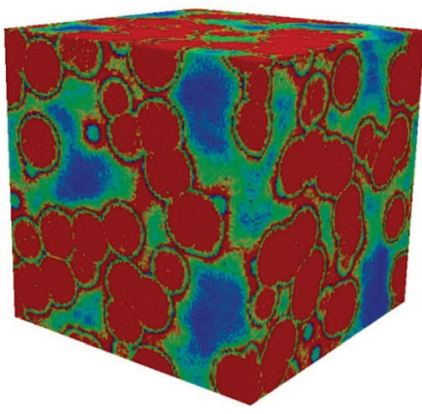

(d) $55 \%$ transformed

Figure 6. Evolution of equivalent plastic strain during phase transformation (homogeneous case). (a) $1 \%$ transformed. (b) $7 \%$ transformed. (c) $20 \%$ transformed. (d) $55 \%$ transformed 


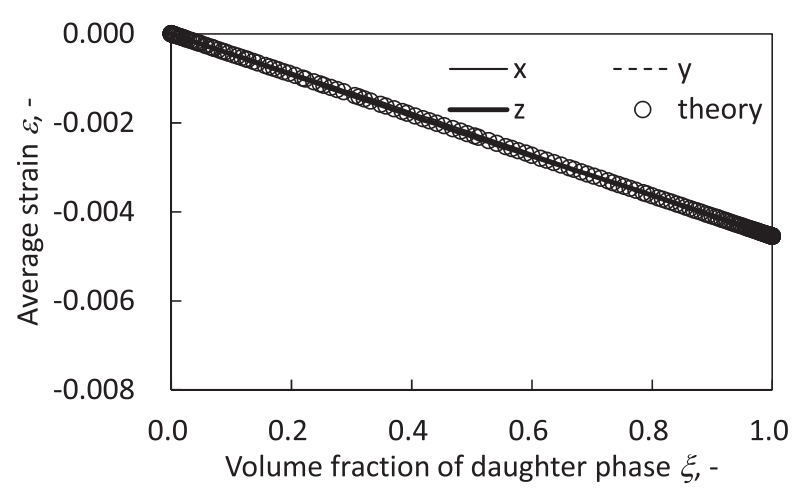

Figure 7. Evolution of strain during phase transformation (heterogeneous case: compared with that by theory).

plastic deformation in the daughter phase to the geometrical relationship with other growing new austenite grains and that is the primary reason behind the effect of heterogeneity in microstructure on anisotropic dilatation during phase transformation.

In the homogeneous case, it has been shown that the overall plastic strain should theoretically be null after Leblond et al. [8]. As a consequence, the exact macroscopic transformation strain of the polycrystal reads

$$
\bar{\varepsilon}^{m}=\beta \zeta .
$$

where $\bar{\varepsilon}^{m}$ denotes the macroscopic transformation strain (isotropic), $\beta$ is the total transformation strain and $\zeta$ is the volume fraction of the transformed phase. Calculation results by Equation (22) are compared with those by numerical approach for the homogeneous case are shown in Figure 7. It is clearly shown that the macroscopic transformation strain during phase transformation is isotropic and which coincides well with the theory. This supports the interpretation of isotropic transformation strain by homogeneous microstructure appeared in the experiment on Part 1 and literature [5]. Moreover, both calculations with heterogeneous and homogeneous microstructure use exactly the same constitutive models and parameters other than the microstructure. This implies the significant effect of microstructure on the total transformation strain.

\section{Conclusion}

A CPFFT model has been established reproducing mechanical simulation incorporating microscopic transformation strain which in turn results in a macroscopic plastic strain in two distinct microstructures; heterogeneous microstructure with pearlite band structure and homogeneous one. The postulate of the geometrical constraint of band structure made in Part 1 was confirmed with the proposed numerical model and the anisotropic transformation strain (significant thickness reduction) with band structure under $\alpha \rightarrow \gamma$ phase transformation was well reproduced.

\section{Disclosure statement}

No potential conflict of interest was reported by the authors.

\section{ORCID}

Takayuki Otsuka (D) http://orcid.org/0000-0003-4326-7357

Kazuo Okamura (D) http://orcid.org/0000-0001-5356-1619

Brigitte Bacroix (D) http://orcid.org/0000-0001-6138-9935

\section{References}

[1] Inoue T, Wang ZG, Miyao K. simulation of quenching process of carburized steel gear wheel under metallothermo-mechanical coupling Elsevier science publishers, Paris, B. V. North-Holland. Proc. IUTAM Thermomec. Coupling in Solids, 1987-5:257-262.

[2] Ju D Y, Zhang W M, Zhang Y. Modeling and experimental verification of martensitic transformation plastic behavior in carbon steel for quenching process. Mater Sci Eng A. 2006;438-440:246-250. Heat Treating 2000: Proc. of the 20th Conference (ASM International). 2000.

[3] Montalvo-Urquizo J, Liu Q, Schmidt A. Simulation of quenching involved in induction hardening including mechanical effects. Comp Mater Sci. 2013;79:639-649.

[4] Inoue T, Okamura K. Material Database for Simulation of Metallo-Thermo-Mechanical Field.

[5] Kop TA, Sietsma J, van der Zwaag S. Anisotropic dilatation behaviour during transformation of hot rolled steels showing banded structure. Mater Sci Technol. 2001;17:1569-1574.

[6] Siwecki T, Koziel T, Hutchinson B, et al. Effect of microsegregation on phase transformation and residual stress. Mater Sci Forum. 2007;539-543:4596-4601.

[7] Jaramillo RA, Lusk MT, Mataya MC. Dimensional anisotropy during phase transformations in a chemically banded 5140 steel. part I: experimental investigation. Acta Mater. 2004;52:851-858.

[8] Leblond JB, Mottet G, Devaux DC. A theoretical and numerical approach to the plastic behaviour of steels during phase transformations-I. derivation of general relations. J Mech Phys Solid. 1986;34(4):395-409.

[9] Barbe F, Quey R, Taleb L. Numerical modelling of the plasticity induced during diffusive transformation. Case of a cubic array of nuclei. Eur J Mech A Solid. 2007;26:611-625.

[10] Barbe F, Quey R, Taleb L, et al. Numerical modelling of the plasticity induced during diffusive transformation. An ensemble averaging approach for the case of random arrays of nuclei. Eur J Mech A Solids. 2008;27:1121-1139.

[11] Barbe F, Quey R. A numerical modelling of 3D polycrystal-to-polycarystal diffusive phase transformations involving crystal plasticity. Int J Plast. 2011;27: 823-840.

[12] Connolly DS, Kohar CP, Mishra RK, et al. A new coupled thermomechanical framework for modeling formability in transformation induced plasticity steels. Int J Plast. 2018;103:39-66.

[13] Mackerle J. Finite element analysis and simulation of quenching and other heat treatment processes. Comput Mater Sci. 2003;27(3):313-332.

[14] Moulinec H, Suquet P. A numerical method for computing the overall response of nonlinear composites with complex microstructure. Comput Methods Appl Mech Eng. 1998;157:69-94. 
[15] Knezevic M, Al-Harbi HF, Kalidindi SR. Crystal plasticity simulations using discrete Fourier transforms. Acta Mater. 2009;57:1777-1784.

[16] Eisenlohr P, Diehl M, Lebensohn RA, et al. A spectral method solution to crystal elasto-viscoplasticity at finite strains. Int J Plast. 2013;46:37-53.

[17] Otsuka T, Brenner R, Bacroix B. FFT-based modelling of transformation plasticity in polycrystalline materials during diffusive phase transformation. Int J Eng Sci. 2018;127:92-113.

[18] Lee S -B, Lebensohn RA, Rollett AD. Modeling the viscoplastic micromechanical response of two-phase materials using Fast Fourier Transfroms. Int J Plast. 2011;27:707-727.

[19] Lebensohn RA, Kanjarla AK, Eisenlohr P. An elastoviscoplastic formulation based on fast Fourier transforms for the prediction of micromechanical fields in polycrystalline materials. Int J Plast. 2012;32-33:59-69.

[20] Asaro RJ, Rice JR. Strain localization in ductile single crystals. J Mech Phys Solid. 1977;25:309-338.

[21] Asaro RJ. Geometrical effects in the inhomogeneous deformation of ductile single crystals. Acta Metall. 1979;27:445-453.

[22] Peirce D, Asaro RJ, Needleman A. An analysis of nonuniform and localized deformation in ductile single crystals. Acta Metall. 1982;30(6):1087-1119.

[23] Peirce D, Asaro J, Needleman A. Material rate dependence and localized deformation in crystalline solids. Acta Metall. 1983;31(12):1951-1976.
[24] Asaro RJ, Needleman A. Texture development and strain hardening in rate dependent polycrystals. Acta Metall. 1985;33(6):923-953.

[25] Hutchinson JW. Bounds and self-consistent estimates for creep of polycrystalline materials. Proc Roy Soc A. 1976;348:101-127.

[26] Pan J, Rice JR. Rate sensitivity of plastic flow and implications for yield-surface vertices. Int J Solids Struct. 1983;19(11):973-987.

[27] Zhang D, Li S. A rate-dependent crystal plasticity analysis of orientation stability in biaxial tension of magnesium. J Mater Sci Technol. 2011;27:175-182.

[28] Kuroda M, Tvergaard V. Shear band development predicted by a non-normality theory of plasticity and comparison to crystal plasticity predictions. Int J Solids Struct. 2001;38:8945-8960.

[29] Hama T, Kuboki A, Takuda H. Crystal-plasticity finiteelement analysis of anisotropic deformation behavior in a commercially pure titanium grade 1 sheet. Int J Plast. 2017;91:77-108.

[30] Hutchinson JW. Elastic-plastic behaviour of polycrystalline metals and composites. Proc R Soc A: Math Phys Eng Sci. 1970;319:247-272.

[31] Greenwood GW, Johnson RH. The deformation of metals under small stresses during phase transformations. Proc R Soc A: Math Phys Eng Sci. 1965;283: 403-422. 